\title{
The majority of A-to-I RNA editing is not required for mammalian homeostasis
}

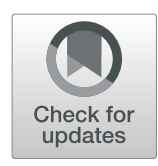

\author{
Alistair M. Chalk ${ }^{1,2}$ (D) Scott Taylor ${ }^{1}$, Jacki E. Heraud-Farlow ${ }^{1,2^{*}+}$ (D) and Carl R. Walkley ${ }^{1,2,3^{*}+}$ (D)
}

\begin{abstract}
Background: Adenosine-to-inosine (A-to-I) RNA editing, mediated by ADAR1 and ADAR2, occurs at tens of thousands to millions of sites across mammalian transcriptomes. A-to-l editing can change the protein coding potential of a transcript and alter RNA splicing, miRNA biology, RNA secondary structure and formation of other RNA species. In vivo, the editing-dependent protein recoding of GRIA2 is the essential function of ADAR2, while ADAR1 editing prevents innate immune sensing of endogenous RNAs by MDA5 in both human and mouse. However, a significant proportion of A-to-l editing sites can be edited by both ADAR1 and ADAR2, particularly within the brain where both are highly expressed. The physiological function(s) of these shared sites, including those evolutionarily conserved, is largely unknown.

Results: To generate completely A-to-l editing-deficient mammals, we crossed the viable rescued ADAR1-editing-

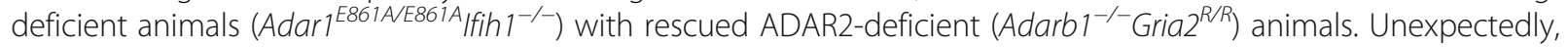

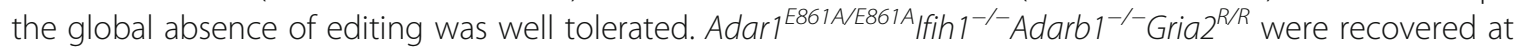
Mendelian ratios and age normally. Detailed transcriptome analysis demonstrated that editing was absent in the brains of the compound mutants and that ADAR1 and ADAR2 have similar editing site preferences and patterns.

Conclusions: We conclude that ADAR1 and ADAR2 are non-redundant and do not compensate for each other's essential functions in vivo. Physiologically essential A-to-l editing comprises a small subset of the editome, and the majority of editing is dispensable for mammalian homeostasis. Moreover, in vivo biologically essential protein recoding mediated by A-to-l editing is an exception in mammals.
\end{abstract}

Keywords: A-to-l editing, ADAR1, ADAR2, RNA editing, Epitranscriptome, RNA modification

\section{Background}

The conversion of adenosine to inosine in RNA (A-to-I RNA editing) is a widespread feature of the transcriptome [1], with tens of thousands of A-to-I sites identified in mouse and millions in human [2-4]. Inosine is interpreted as guanosine upon translation or sequencing, meaning A-to-I editing leads to post-transcriptional Ato-G transitions in RNA. Editing occurs within regions of double-stranded RNA (dsRNA), and inosine has different thermodynamic base pairing properties to adenosine, harboring the potential to alter both the RNA code and the secondary structure [5-8]. A-to-I editing levels vary across transcripts, tissues, and throughout

\footnotetext{
* Correspondence: jhfarlow@svi.edu.au; cwalkley@svi.edu.au

†Jacki E Heraud-Farlow and Carl R Walkley are co-senior authors.

${ }^{1}$ St. Vincent's Institute of Medical Research, 9 Princes St, Fitzroy, VIC 3065,

Australia

Full list of author information is available at the end of the article
}

development ranging from $<1$ to $100 \%$ at any given site $[4,9]$. Editing can occur at specific adenosines within a transcript, termed site-selective editing, or at many sites within an extended region, termed hyperediting or editing enriched regions [3, 10-12]. The vast majority of editing is weak and occurs within repetitive elements (e.g., Alu elements in humans, SINEs in mice). In mice and humans, A-to-I editing is catalyzed by the adenosine deaminase acting on RNA family members ADAR1 (Adar) and ADAR2 (Adarb1). The third mammalian ADAR, ADAR3 (Adarb2), does not have detectable editing activity $[13,14]$. The prevailing view is that siteselective editing is primarily associated with ADAR2, while hyperediting is associated with ADAR1 $[2,4]$.

Altered expression or mutation of ADARs is associated with several human diseases. Loss of function mutations in $A D A R$ causes the infantile encephalopathy Aicardi-Goutières syndrome (AGS) [15]. AGS patients 
develop a characteristic type I interferonopathy, a transcriptional signature first associated with loss of ADAR1 in the mouse [16, 17]. ADAR1 is overexpressed in a number of cancers which is postulated to contribute to cancer progression and proteome diversity $[18,19]$. Recent work identified a number of cancers to be highly sensitive to loss of ADAR1 and depletion of ADAR1 enhanced activity of immunotherapy [20-22]. Reduced ADAR2 activity and overall editing levels have been reported in central nervous system (CNS) diseases, including amyotrophic lateral sclerosis, autism, and brain cancers $[23,24]$. While the consequences of mutations in the writers of A-to-I editing are clear, the physiological roles and functions of the majority of editing sites are undetermined.

The most striking outcome of A-to-I editing is protein recoding, where editing directly changes the amino acid sequence of the translated protein from that encoded genomically. Recoding of the ADAR2-specific Q/R site in the glutamate receptor Gria2 is essential for post-natal viability in mice [25]. Adarb1 ${ }^{-/-}\left(\right.$Adar2 $\left.^{-/-}\right)$animals die several weeks after birth and were rescued by homozygous single residue A-to-G mutation in the genomic DNA at the edited $\mathrm{Q} / \mathrm{R}$ codon of Gria2, mimicking the constitutive recoding at this site [25]. The Adarb $1^{-/-}$Gria $^{R / R}$ rescued animals are remarkably normal indicating that this single editing site accounts for the lethality, with several subtle phenotypes subsequently reported in the viable rescued animals $[26,27]$. The contributions of the majority of protein recoding events outside of GRIA2 and the reasons for the evolutionary conservation of a subset of them is largely unknown $[28,29]$.

Editing can change splice sites, miRNA binding sites, and pre- and mature miRNAs, as well as alter the production of circular RNAs. However, the vast majority of mammalian editing occurs in repetitive elements/retrotransposons such as short interspersed elements (SINEs) and long interspersed elements (LINEs), including primate restricted $\mathrm{Alu}$ elements, which can form structured long dsRNA [2, 9]. Physiologically, editing by ADAR1 attenuates the immunogenic potential of endogenous dsRNA and prevents an MDA5-mediated innate immune response to self-dsRNA in both human and mouse [30-35]. Adar ${ }^{-1-}\left(\right.$ Adarl $\left.^{-/-}\right)$or editing-deficient $\left(\right.$ Adar1 $\left.^{\text {E861A/E861A }}\right)$ animals die in utero at E11.75-E12.5 [36, 37] and E13.5 [30], respectively, which can be rescued by loss of the cytosolic dsRNA sensor MDA5 (encoded by Ifih1) or its downstream effector MAVS [30-32]. The same genetic pathway is present in human ADAR1-deficient cell lines, with the MDA5/MAVS axis being the principal physiological sensor of unedited endogenous dsRNA [31, 34]. This demonstrates a conserved mammalian response to unedited RNA that is not dependent on primate restricted $A l u$ elements. The requirement for editing by Adar1 appears to be distinct to that of ADAR2, and essential ADAR1-dependent protein recoding events, analogous to ADAR2/Gria2, have not been identified [38].

Our understanding of the in vivo functions of A-to-I editing in mammals is incomplete. There are detailed maps of the numbers, levels, and tissue distribution of A-to-I editing across multiple species [4]. It is known that ADAR1 and ADAR2 share similar sequence neighbor preferences around the edited adenosine [39] and can compensate for each other on many endogenous substrates or when directed for programmable editing [40-42]. Compensatory editing has the potential to mask physiologically important phenotypes and functions of A-to-I editing in the respective single mutant models. Moreover, with tens of thousands of editing events occurring in vivo during murine development and aging, the contribution of these has not been determined outside of a small number of targets. To address this, we have now generated and assessed compound editingdeficient Adar1 ${ }^{\text {E861A/E861A }}$ Adarb1 $^{-/-}$null mice also containing the respective Ifih $1^{-/-}$and $G r i a 2^{R / R}$ suppressor alleles. We show here that mice completely lacking A-to-I editing were recovered at Mendelian ratio. Although half of editing sites in the brain were shared by ADAR1 and ADAR2, including many that are evolutionarily conserved and within coding regions, mice completely lacking A-to-I editing developed and aged normally. This demonstrates that ADAR1 and ADAR2 do not physiologically compensate by editing additional shared essential sites in vivo.

\section{Results}

A-to-I editing-deficient animals are recovered the expected frequency and age normally

To generate completely A-to-I editing-deficient mammals, we crossed the viable rescued ADAR1-editing-deficient ani-

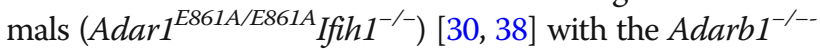
Gria2 $^{R / R}$ animals [25]. From these crosses, we recovered all expected genotypes and assessed their long-term survival (Fig. 1a-e). Surprisingly, completely editing-deficient

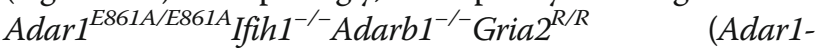
${ }^{E 861 A / E 861 A} A_{\text {darb }}{ }^{-/}$) animals were born at the expected Mendelian frequency (Fig. 1a, b) and long-term survival percentages were not significantly different to controls within the available sample size (Fig. 1c-e, Additional file 1: Figure S1). Under standard housing conditions, the Adar1E861A/E861A Adarb1 $^{-/-}$animals have survived $>1$ year of age to date ( $n=15>52$ weeks old; $n=2>80$ weeks). Furthermore, the Adarb $1^{-/-} \mathrm{Gria}^{R /+}$ genotype had reduced postnatal survival consistent with their original description [25] and this was not worsened by the further loss of ADAR1 editing (Fig. 1c, d). This indicates that ADAR1 and ADAR2 are non-redundant during mouse development and 
A.

\begin{tabular}{|c|c|c|}
\hline \multicolumn{3}{|c|}{ 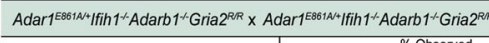 } \\
\hline Genotype & Observed & $\begin{array}{l}\% \text { Oobserved } \\
(\% \text { Expected) }\end{array}$ \\
\hline 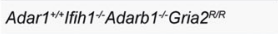 & 11 & $17.7 \%(25 \%)$ \\
\hline 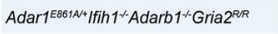 & 35 & $56.4 \%(50 \%)$ \\
\hline 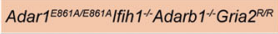 & 16 & $25.8 \%(25 \%)$ \\
\hline al (number genot) & 62 & 9 litte \\
\hline
\end{tabular}

B.

\begin{tabular}{|c|c|c|}
\hline Genotype & Observed & $\begin{array}{l}\% \text { Observed } \\
\text { (\% Expected) }\end{array}$ \\
\hline 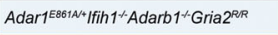 & 29 & $45.3 \%(50 \%)$ \\
\hline 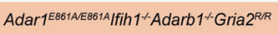 & 35 & $54.7 \%(50 \%)$ \\
\hline Total (number genotyped) & 62 & (11 litters) \\
\hline
\end{tabular}

c.

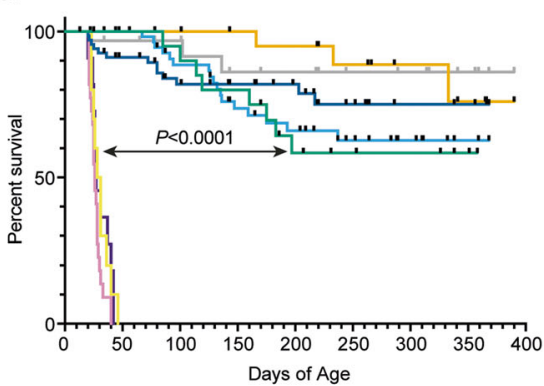

D.

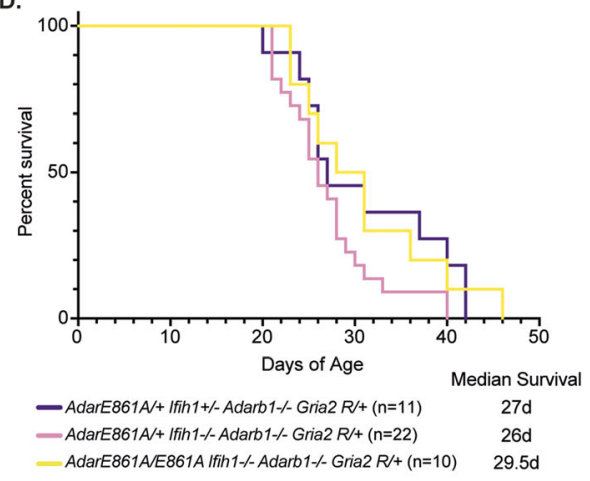

E.

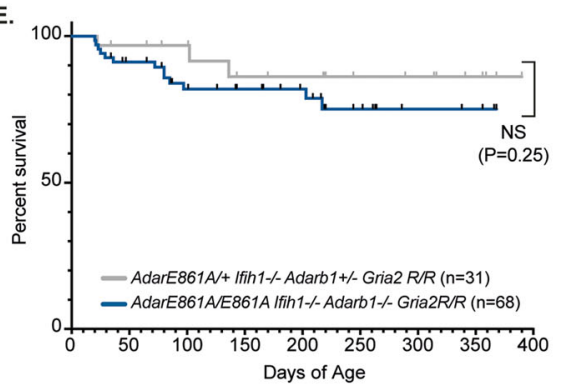

F.
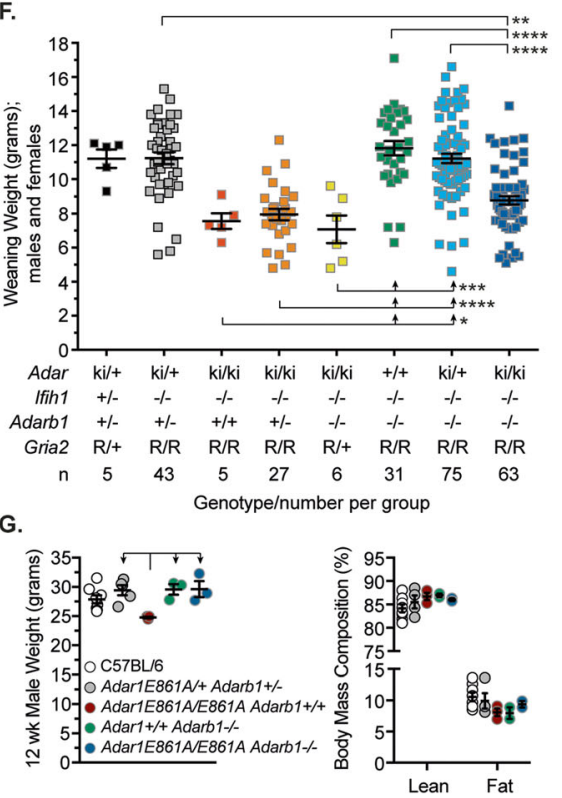

Fig. 1 A-to-l editing-deficient mice are viable with a normal lifespan. a Breeding data from intercrosses of Adar1 $1^{E 861 A++} \mid f i h 1^{-1-} A d a r b 1^{-/-} G r i a 2^{R / R}$ males

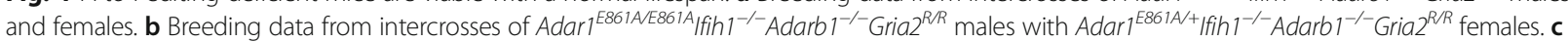
Survival data for all genotypes; numbers per genotype and statistical comparison across all genotypes (pairwise log-rank (Mantel-Cox) test); $P$ value as indicated or ${ }^{* * *} P<0.001$. d Comparison of survival of Adarb $1^{-1-} \mathrm{Gria}^{R /+}$ animals with either heterozygous Adarl ${ }^{\text {E661A+t }}$ (purple and pink lines) or

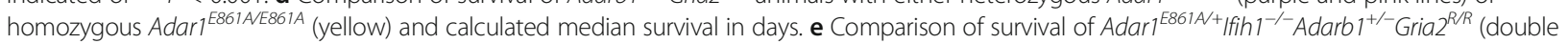

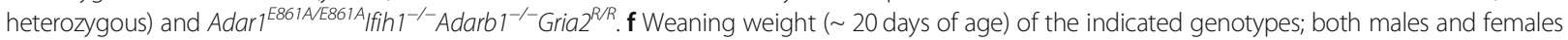
included. ${ }^{*} P<0.05,{ }^{*} P<0.01,{ }^{* *} P<0.001$ (ordinary one-way ANOVA with multiple comparisons correction (Tukey's)). g Body weight and body mass

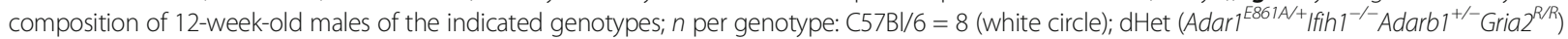

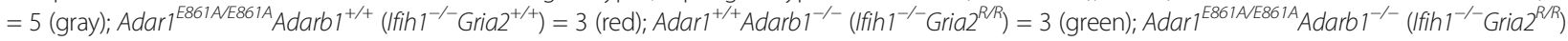
$=3$ (blue) ${ }^{*} P<0.05$ (ordinary one-way ANOVA with multiple comparisons correction (Tukey's)) 
lifespan. Strikingly, it also demonstrates that the single adenosine at the Q/R site of Gria2 represents the only physiologically essential protein recoding event in vivo.

At weaning, Adar ${ }^{E 861 A / E 861 A} I f i h 1^{-/-}$animals are smaller than controls [38], irrespective of the Adarb1/ Gria2 genotype, and this was maintained in Adar1${ }_{\text {E861A/E861A }}$ ddarb1 $^{-/}$animals (Fig. 1f). We compared cohorts of adult males from the indicated genotypes to age-/facility-matched C57BL/6 animals. Adult Adar1E861A/E861A Ifih1 $1^{-/-}$animals weighed less than all other genotypes but had a normal body mass composition (Fig. 1g). In contrast, all other genotypes had a normal body weight and body mass composition compared to both C57BL/6 and Adar1 ${ }^{E 861 A /{ }^{+}}$Ifih1 $^{-/-}$Adarb1 $^{+/--}$ $\mathrm{Gria}^{R / R}$ (double heterozygous; dHet) (Fig. 1g). Therefore, loss of ADAR2 does not modify the initial postnatal runting of Adar1 ${ }^{E 861 A / E 861 A}$ Ifih $1^{-/-}$animals but does enable recovery to normal weight by 12 weeks of age. As hematopoietic cells in the mouse are particularly sensitive to ADAR1 loss, analysis of the hematopoietic populations in the peripheral blood and other hematopoietic organs was performed (Fig. 2). The Adar1 ${ }^{E 861 A / E 861 A}$ Adarb1 $^{-/-}$were not significantly different to editing-proficient controls (Fig. 2) [38]. A genotype blinded histological assessment of 20-25week-old male Adar1 ${ }^{E 861 A / E 861 A} A d a r b 1^{-/-}$animals and dHets did not find any significant difference between genotypes (Additional file 2: Dataset S1). Therefore, once self-sensing of unedited dsRNA is prevented by loss of MDA5 and the single GRIA2 Q/R site is genomically reinstated, the complete absence of A-to-I editing in vivo is well tolerated. This demonstrates that there are no essential roles of ADAR1 and ADAR2-mediated editing that are compensated for in the single mutants by the other homolog.

\section{ADAR2 loss does not modify ADAR1-dependent transcriptional signatures.}

RNA-seq was performed on the whole brain from 12-week-old males to assess changes in gene expression. The brain was chosen as it expresses robust levels of both ADAR1 and ADAR2, unlike many peripheral tissues [38]. For comparison, we included a prior dataset from 12-week-old male $\operatorname{Adarl}^{+/+}{ }^{2}$ fih1 $^{-/-}$and Adar1 ${ }^{E 861 A / E 861 A}$ Ifih1 $^{-/-}$whole brain (Fig. 3a) [38]. Differential gene expression analysis

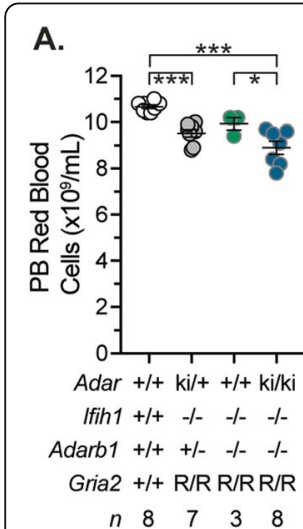

F.

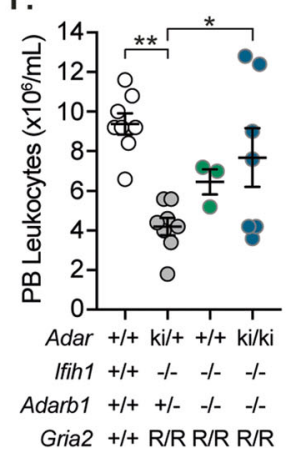

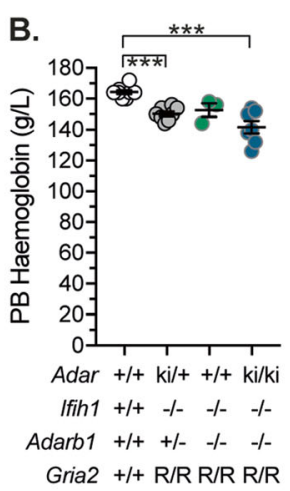
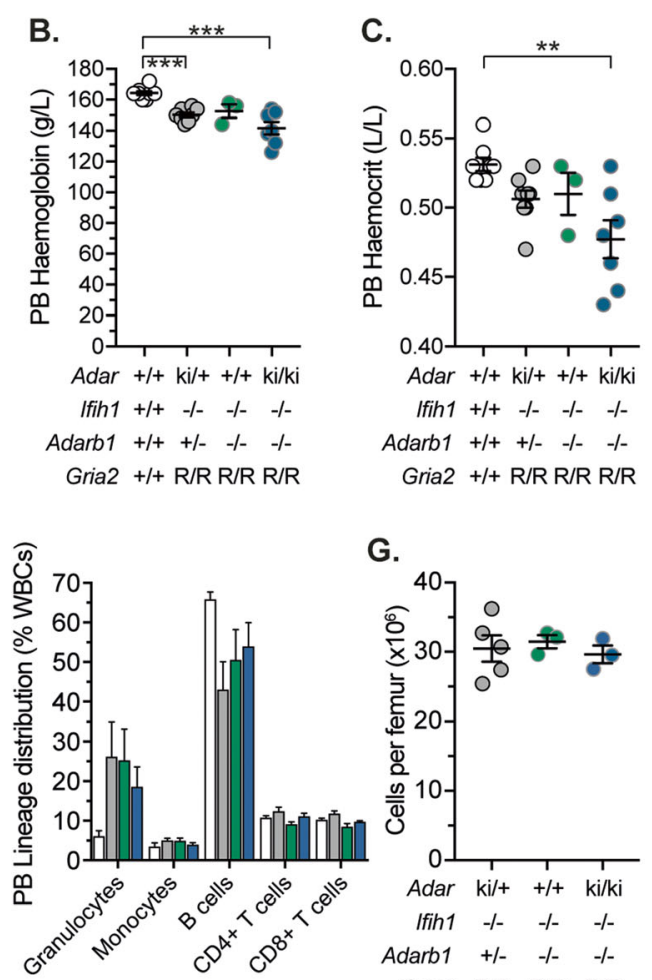

G.
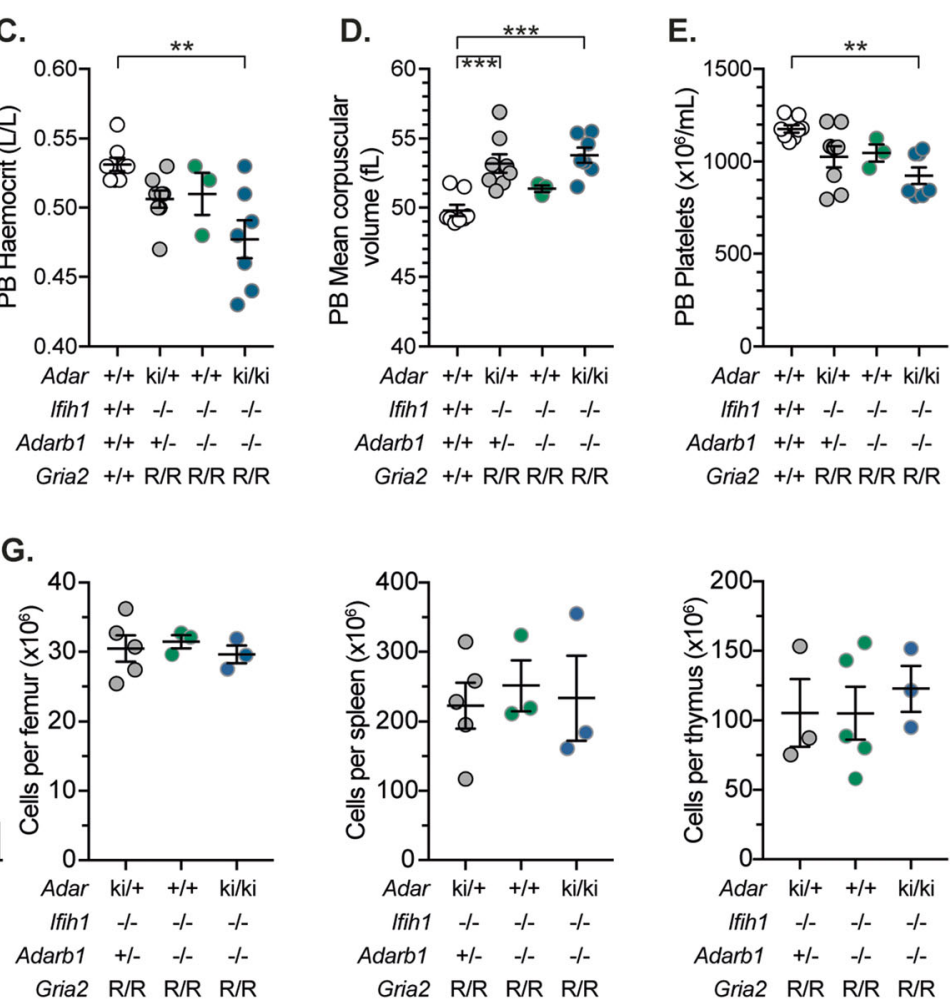

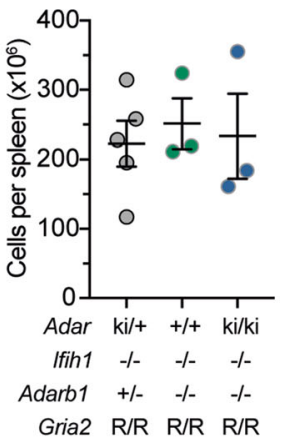

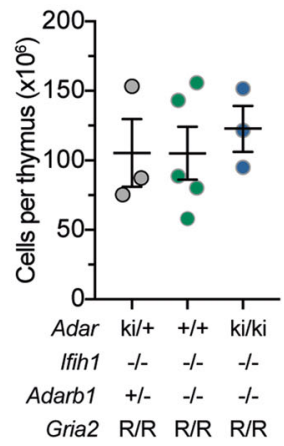

Fig. 2 Peripheral blood and hematopoietic parameters of Adar1 ${ }^{E 861 A E E 861 A} A d a r b 1^{-1-}$ mice. a Red blood cell counts. b Hemoglobin. $\mathbf{c}$ Hematocrit. $\mathbf{d}$ Mean corpuscular volume. e Platelet count. $\mathbf{f}$ Peripheral blood leukocyte numbers and lineage distribution from the indicated genotypes, C57BI/6

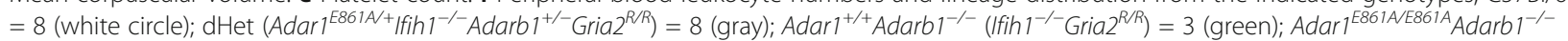
$\left(\right.$ Ifih $1^{-1-}$ Gria $\left.2^{R / R}\right)=7$ (blue), ${ }^{*} P<0.05,{ }^{* *} P<0.01$. g Cellularity of the femurs, spleen, and thymus from the indicated genotypes, $n=3$ per genotype. All counts were performed on peripheral blood from 12-18-week-old male animals of the indicated genotypes. Number of animals in each genotype indicated in panel a. Statistical analysis: one-way ANOVA with correction for multiple comparisons; ${ }^{*} P<0.05 ;{ }^{* *} P<0.01 ;{ }^{* * *} P<0.001$ 
A.

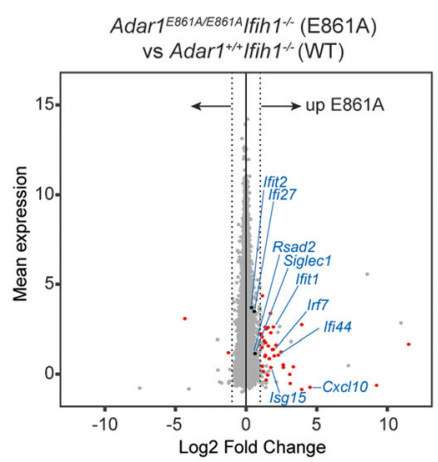

C. Adar1 1861AE861Alfih1-Adarb1-Gria2RR(dKO)

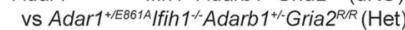
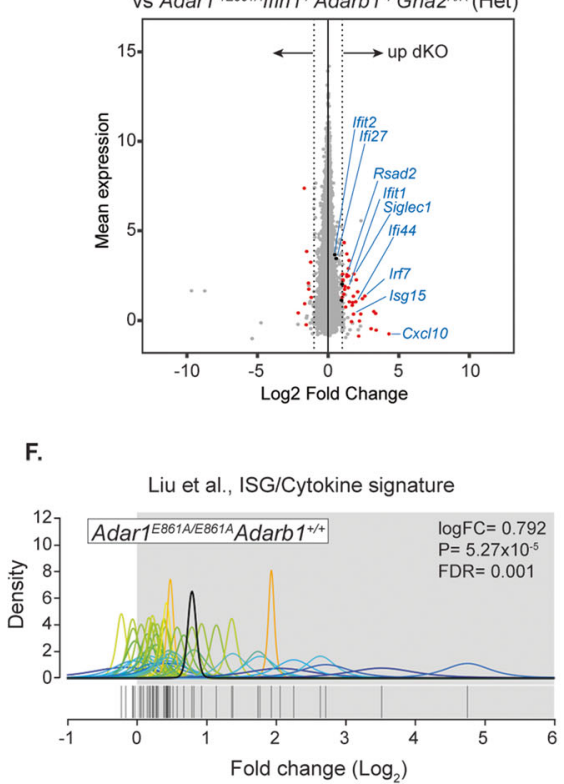

B.

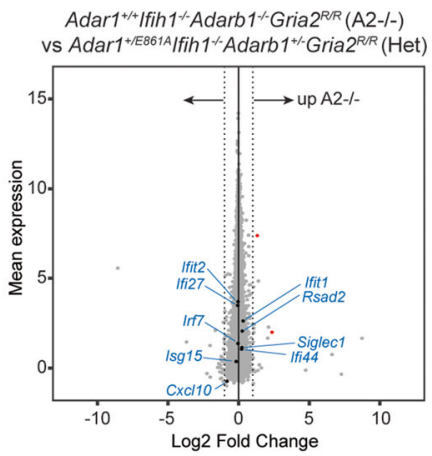

D.

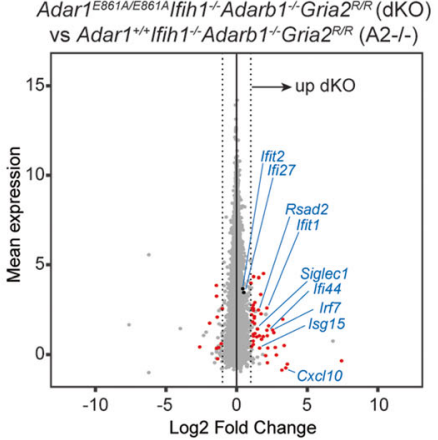

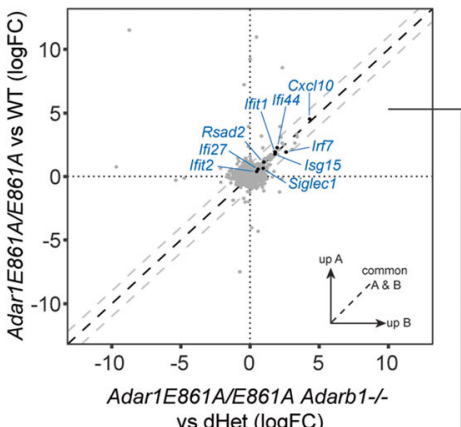

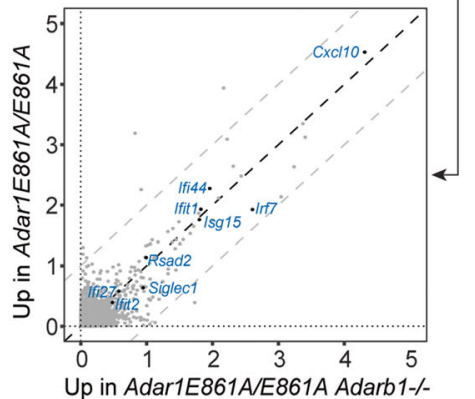

Fig. 3 ADAR2 loss does not modify the transcriptional signature associated with the loss of Adar1-mediated RNA editing. Analysis of differential

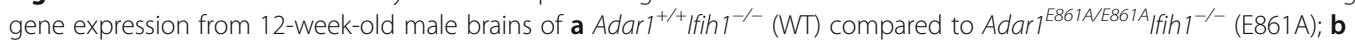

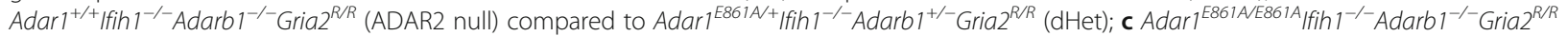

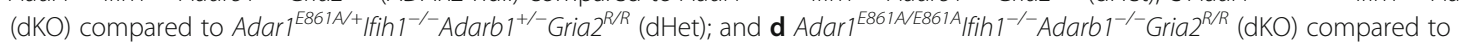
Adar $^{+/+} \mid$fih $^{1^{-/}}$Adarb $^{-/-}$Gria2 $^{R / R}\left(\right.$ Adar2 $^{-/}$); $n=3$ per genotype; red indicated FDR $<0.05$. e Comparison of the differential gene expression signatures of the E861A (a) and $\mathrm{dKO}$ (c) samples. The increased expression of the transcripts highlighted in blue is shared between murine and human ADAR1 mutants. Top panel: $y$-axis has the gene expression comparison of the Adar1 ${ }^{\text {E661AVE861A }}$ VS WT; $x$-axis has the gene expression

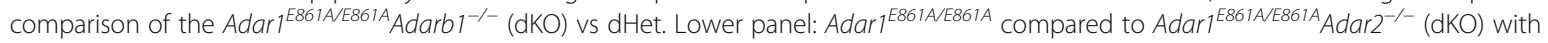
expanded view of the upper right quadrant. f QuSAGE pathway analysis of the consensus interferon-stimulated gene (ISG)/cytokine signature

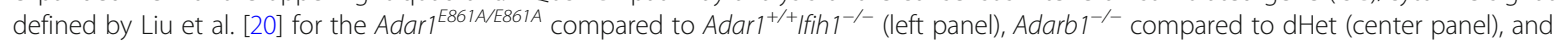

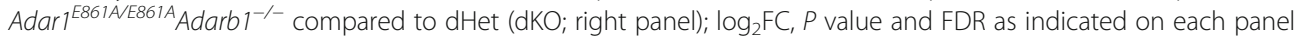

demonstrated that the most significant changes occurred in the Adar1 ${ }^{\text {E861A/E861A Ifih1 }}{ }^{-/-}$and Adar1${ }_{\text {E861A/E861A }}$ Adarb1 $^{-/-}$genotypes where a modest activation of the innate immune/interferon signature was present, including the genes used diagnostically in humans with ADAR1 mutation (Fig. 3a-e, Additional file 1: Figure S1, Additional file 3: Dataset S2) $(15,20,38)$. The loss of ADAR2 editing alone did not significantly impact gene expression (Fig. 3d, e). Notably, the loss of both ADAR1 and ADAR2 editing did not significantly modify gene expression outside of that dependent on the loss of ADAR1 editing (Fig. 3e). Therefore, A-to-I editing is not required for homeostatic gene expression or regulation of gene expression and its absence does not significantly alter the brain transcriptome. Pathway analysis demonstrated that the modest activation of the ISG/cytokine signature is unique to ADAR1editing-deficient samples, even in the absence of MDA5, and that the loss of ADAR2 does not further exacerbate it (Fig. 3f) [20]. 
Transcriptome-wide loss of A-to-I editing is tolerated Sanger sequencing of a well-characterized substrate, $H t r 2 c$, from the 12-week-old male brains confirmed the complete loss of editing at sites A and B in Adar1$E 861 A / E 861 A$ samples, the specific loss of site D from
Adar2 $2^{-/-}$, and the absence of editing at all sites from the Adar1 ${ }^{\text {E861A/E861A }}$ Adarb1 $^{-/-}$(Fig. 4a) [37]. To understand the ADAR1- and ADAR2-dependent editome, we compared the editing sites that were present, absent, or gained in each genotype against a database

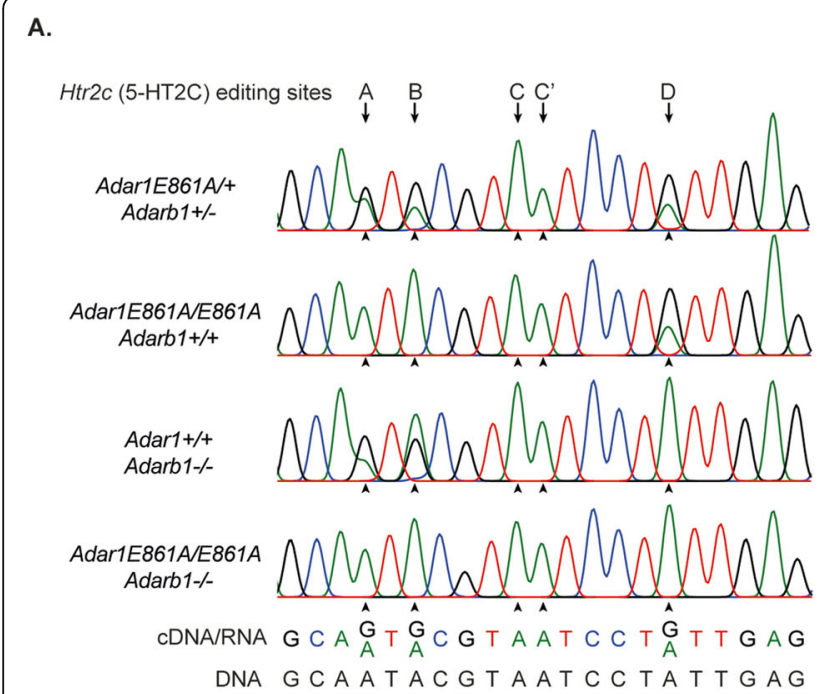

c.

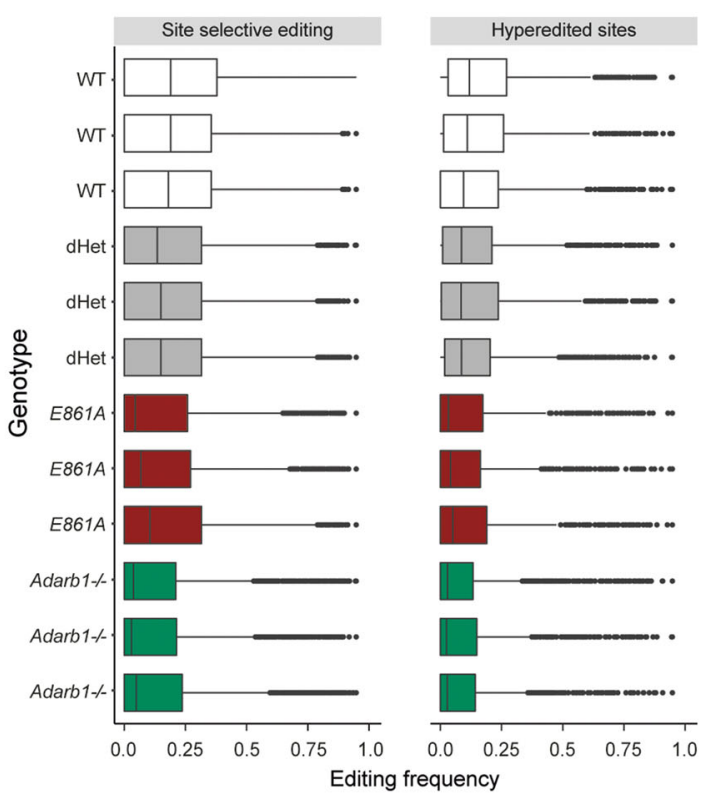

B.
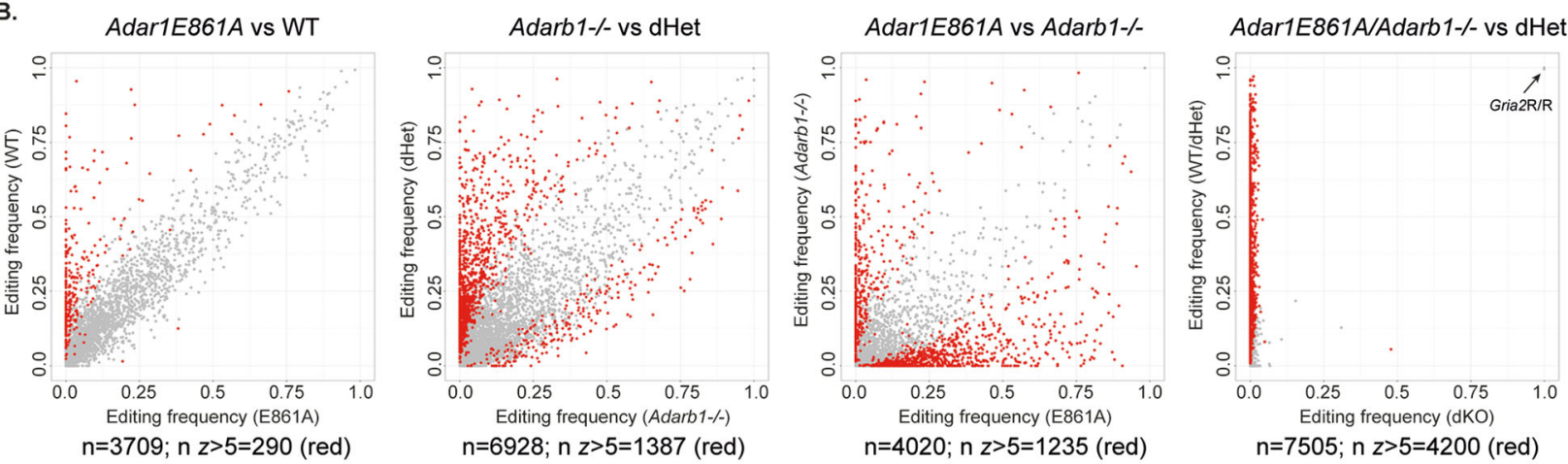

D.
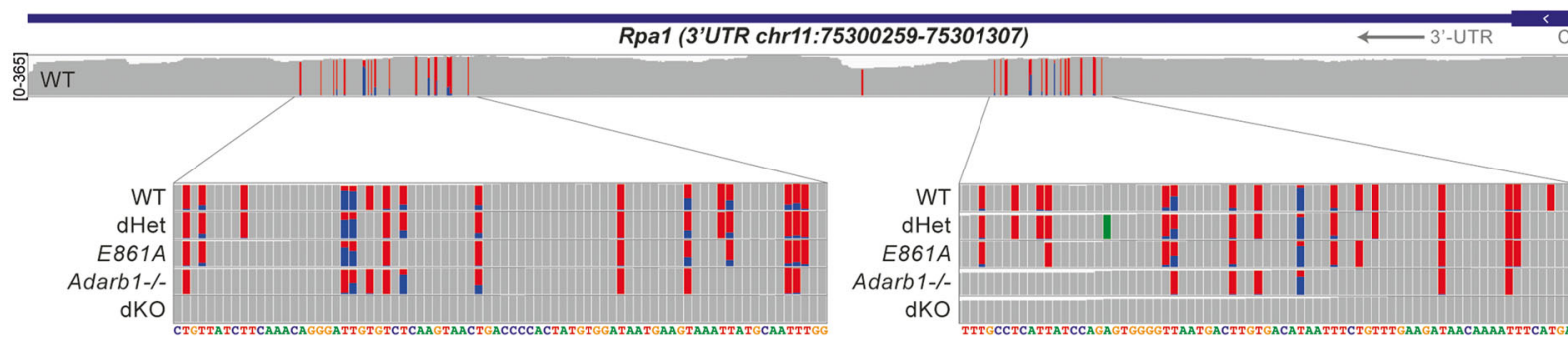

Fig. 4 Adari ${ }^{E 861 A / E 861 A}$ Adarb $1^{-1-}$ have lost A-to-l editing across the transcriptome. a Analysis of A-to-l editing of the Htr2C receptor at the known sites A-D by Sanger sequencing. Genotypes as indicated. b Analysis of editing sites across the genotypes. A dataset of 57,077 murine editing sites was compiled and the datasets assessed for editing at these sites. Sites required $\geq 50$ read coverage and an editing rate of $\geq 0.01$ ( $\geq 1 \%$ ) to be included. The number that passed this threshold for each comparison is listed, and the numbers that are significantly different based on the $z$ factor ( $z \geq 5$; Jacusa analysis method) are indicated in red. c Editing frequency across coding/site-selective and repetitive/hyperediting sites in the transcriptome in the individual samples from the WT, dHet, Adar ${ }^{\text {E861AEE } 861 \mathrm{~A}}$, and Adarb $1^{-1-}$. Sites required $\geq 50$ read coverage and an editing rate of $\geq 0.01(\geq 1 \%)$ to be considered. Boxplot represents the $25 \%$ quantile to $75 \%$ quantile with the median indicated. $\mathbf{d}$ Editing of the 3 'UTR of Rpa1 transcript in each of the indicated genotypes 
of 57,077 murine editing sites compiled from published databases (RADAR [8]), previous publications [4, 30, 43], and unpublished murine datasets (JH-F, AMC, and CRW). Analyses of known editing sites demonstrated that a significant proportion could be edited by either ADAR1 or ADAR2 (Fig. 4b), with subsets being ADAR1- or ADAR2-specific (sites clustering on the $y$-axis in the first or second panel, respectively). As anticipated, A-to-I edit-

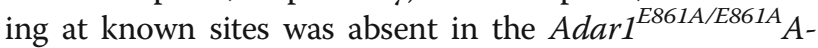
darb1 $^{-1-}$ samples (Fig. 4b; Additional file 1: Figure S3). The loss of A-to-I editing in the double mutant confirmed that there are no alternative enzymes capable of this modification in mouse. Surprisingly, the loss of either ADAR1 or ADAR2 individually had a largely comparable effect on the transcriptome-wide distribution and levels of editing at either site-selective or repetitive/hyperedited regions (hyperediting defined as 10 or more editing sites per 100 bp; Fig. 4c). As an example, Rpa1 was edited by both ADAR1 and ADAR2 (Fig. 4d). Evolutionarily conserved editing events, except the genomically engineered Gria2 $2^{R / R}$, were absent in the Adar1 ${ }^{E 861 A / E 861 A}{ }^{A d a r b 1} 1^{-/-}$ samples indicating that the global absence of protein recoding does not have a pathogenic effect in vivo, under standard housing conditions (Fig. 5a).

\section{ADAR1 and ADAR2 do not have unique substrate preferences}

Using datasets from all genotypes, we investigated the characteristics of ADAR1- and ADAR2-specific editing events. While from a single tissue and developmental timepoint these datasets are genetically controlled, providing high confidence with which to delineate the characteristics of these sites. When evolutionarily conserved sites were assessed, the majority were either able to be edited equivalently by either ADAR1 or ADAR2 or were ADAR2-specific events. A small number of the conserved sites were ADAR1-specific, and another subset showed a pattern of editing suggesting inhibition of specific editing by the alternative ADAR (Fig. 5a). Such a phenomenon has previously been reported at selected targets in mouse [44] and when assessed transcriptomewide in Caenorhabditis elegans [45]. We then defined the genotype-specific editing events from the entire dataset and assessed the characteristics of these sites. ADAR1-specific and ADAR2-specific sites shared a similar overall number and location/distribution across the transcript (Fig. 5b). Editing of B1 and B2 repeat elements was largely comparable between ADAR1 and ADAR2. When the editing frequency was assessed, ADAR1specific editing events within B2 elements were edited to a lower average level that the average editing frequency of all sites in B2 elements; however, there was no significant difference between ADAR1 and ADAR2 (Fig. 5c). Despite the single mutant phenotypes, there was no clear preference toward editing of repetitive elements by ADAR1 nor toward site-selective/recoding for ADAR2 (Fig. 5b). Although ADAR2 was responsible for a larger proportion of the conserved editing sites (Fig. 5a), analysis of the sequence context of the edited adenosine did not reveal a strong sequence motif in neighboring nucleotides or difference between ADAR enzymes, consistent with a lack of sequence specificity by dsRNA binding proteins in general (Fig. 5b). The direct comparison of the ADAR1- and ADAR2-specific events did not demonstrate significant differences in editing level by location (Fig. 5c). A recently reported independent analysis of editing sites in a range of ADAR mutant mice also concluded that both ADAR1 and ADAR2 have similar activity and efficiency for both repetitive regions and site-selective editing in vivo [46]. Using definitive genetic controls, we conclude that ADAR1 and ADAR2 have similar editing site distributions and that the sequence context of the edited adenosine does not account for the editing specificity. Cell type-specific expression patterns and cellular localization of ADAR1 and ADAR2, RNA structure, splicing efficiency, cis-regulatory elements, or other RNA binding factors may be additional determinants of ADAR specificity [4, 28, 46, 47].

\section{Discussion}

A-to-I editing in the mouse occurs at tens of thousands of sites in both coding and non-coding regions and is developmentally dynamic. Advances in transcriptome sequencing have greatly increased our understanding of the breadth and extent of A-to-I editing across evolution and within a given species. It is now apparent that A-toI editing is a highly prevalent epitranscriptomic mark in mammals and that it has the potential to influence many important processes including miRNA sequence and biogenesis, miRNA binding sites, RNA splicing, circular RNA biogenesis, and allowing tolerance to repetitive elements and dsRNA structure. While evidence exists for roles of A-to-I editing in all of these processes, the physiological function of the majority of editing sites is unknown.

Mutants of the individual ADAR's have been reported in the mouse and have provided important insight into the physiological roles of these enzymes. These studies demonstrated that ADAR2 was essential for protein recoding editing [25], particularly in the central nervous system, while ADAR1 was required to prevent sensing of endogenous RNA by MDA5 [3]. These genetic studies have focused attention on a small subset of the tens of thousands of sites potentially edited in the mouse transcriptome during development and aging. The extent of functional redundancy between ADAR1 and ADAR2 to mask important physiological roles of Ato-I editing was unknown, potentially preventing 

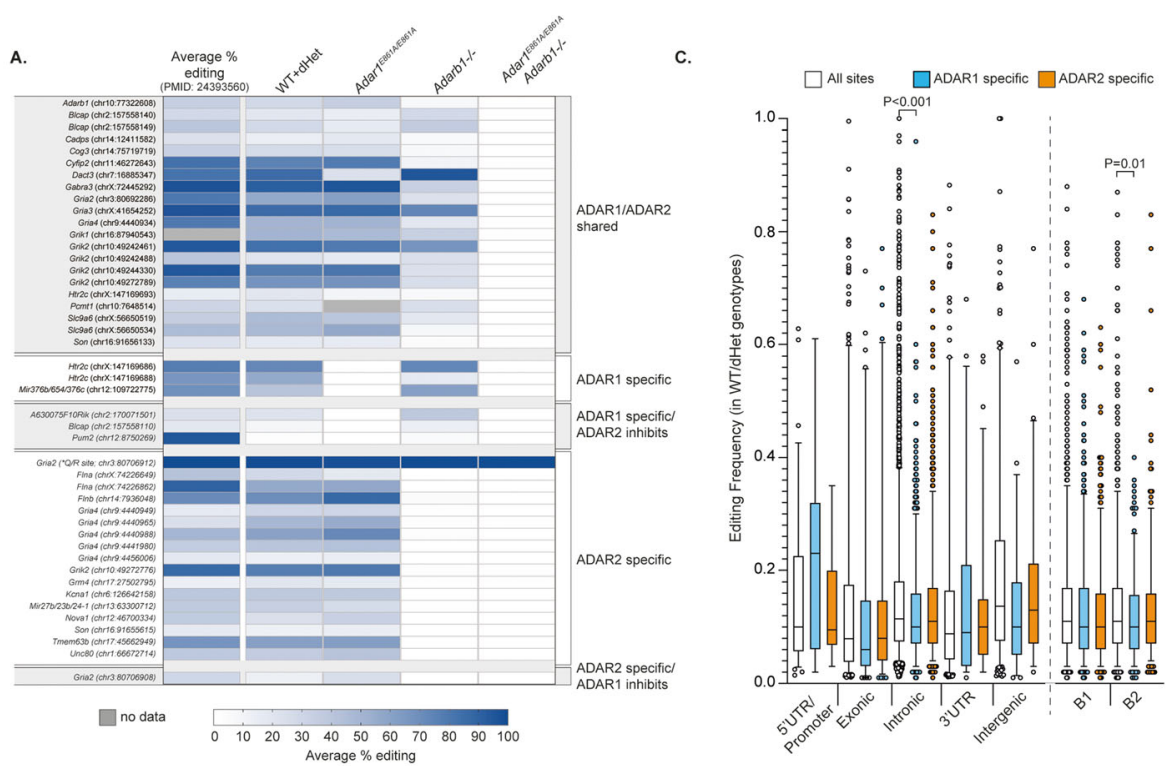

B.

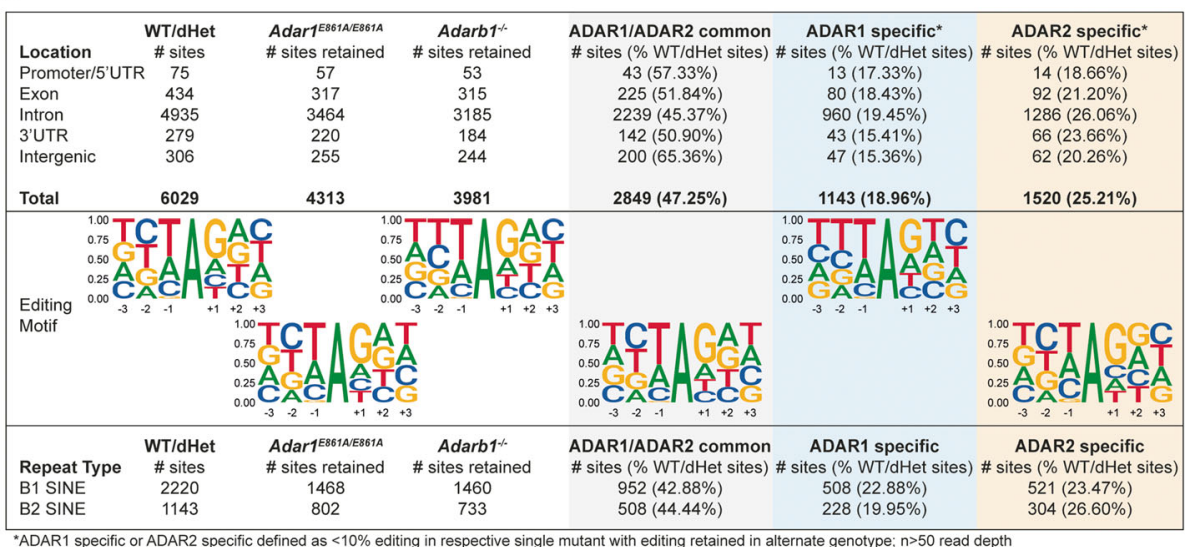

Fig. 5 ADAR1- and ADAR2-specific sites are comparable. a Analysis of evolutionarily conserved A-to-l editing events across the genotypes. Average editing for each site was calculated plotted with reference to the levels at each site identified by Pinto et al. [29]. ADAR1/ADAR2 shared sites are defined as having $>10 \%$ and $<150 \%$ editing compared to the average editing rate of the WT and Adar $7^{E 861 \mathrm{~A}+}{ }^{A}$ Adarb $1^{+/}$(dHet) samples combined (WT+dHet); ADAR1-specific sites have $<10 \%$ editing of this site in the Adar $7^{\text {E861AEE }}{ }^{261 A}$ samples and unchanged editing in the Adarb $^{-1-}$ compared to WT+dHet; ADAR1-specific/ADAR2 inhibits sites have $<10 \%$ editing of this site in the Adar1 ${ }^{\text {EB61AEE E } 61 A}$ samples and $>150 \%$ editing of WT+dHet levels in the Adarb $1^{-1-}$; ADAR2-specific sites have $<10 \%$ editing of this site in the Adarb $1^{-1-}$ samples and unchanged editing in the Adar1 $1^{\text {E66IAEE861A }}$ compared to WT+dHet; ADAR2-specific/ADAR1 inhibits sites have $<10 \%$ editing of this site in the Adarb $1^{-/-}$samples and $>$

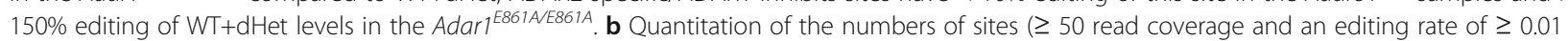
$(\geq 1 \%)$ and genomic location across genotypes. ADAR1- or ADAR2-specific sites were defined as having $<10 \%$ editing of a site in the one genotype and retained editing in the alternative genotype. The percentage of sites that are ADAR1 or ADAR2 specific is indicated in brackets as a percent of the total number of sites for each location. The sequence context of the editing sites for each classification was derived with Seqlogo. The distribution of editing in B1 and B2 SINEs was mapped from the total sites identified in each genotype. c The genomic distribution/repeat type and average editing level for the ADAR1 and ADAR2 sites compared to the all sites observed in the control (WT+dHet genotype combined). Box and whiskers plot with 5-95 percentile shown. No significant difference between genotypes or $P$ value as indicated (ANOVA with multiple comparisons correction)

appreciation of meaningful consequences of editing. The complete absence of editing causes phenotypic consequences in both C. elegans (chemotaxis) $[48,49]$ and Drosophila (staggering phenotype [50]); however, both species lack an ADAR1 homolog which means the functions of the many sites edited by both ADAR1 and ADAR2 in mammals are not able to be understood [51, 52]. Through interbreeding of the rescued ADAR1-editing-deficient mice and the $A d a r b 1^{-1-}$ Gria2 $^{R / R}$ animals, we were able to evaluate the organismal requirement for A-to-I editing in mammalian development and homeostasis.

Historically, editing has been most prominently linked to protein recoding, where editing of adenosines within exonic regions changes the genomically encoded amino 
acid resulting in a protein with altered sequence and, potentially, function. This type of A-to-I editing, particularly in the nervous system, has demonstrated physiologically functional consequences across species $[25,50,53,54]$. The paradigm for recoding editing remains GRIA2 [25, 55]. More recently, analysis of additional conserved recoding sites including FLNA, NEIL1, and AZIN1 has been reported. The deletion of the conserved editing complementary sequence (ECS) from Filamin A (FLNA) leads to a loss in editing and prevents a Q2341R amino acid change in the mouse. This site is highly edited in both human and mouse cardiovascular tissues and arteries [56]. FLNA ${ }^{\triangle \mathrm{ECS}}$ mice had no apparent abnormalities and normal life expectancy and fertility. However, both isolated aortae and vascular smooth muscle cells had altered function in vitro, and the mice had a mild diastolic hypertension at rest and altered arterial and cardiac remodeling [56]. The editing of NEIL1, a protein implicated in the DNA damage response, leads to a lysine to arginine (K242R) substitution. The two protein forms (unedited K242 and edited R242) have different binding kinetics and affinities for DNA substrates [57]. An increased editing of AZIN1 was identified in hepatocellular carcinoma [58]. The editing of AZIN1 resulted in an S367G recoding event, leading to altered cellular localization of the edited protein and proposed gain-of-function activity. Such studies provide evidence that protein recoding can have specific in vivo functions, demonstrating that these can be important but not essential for viability. It is worth considering that with the exception of the recent in vivo model of FLNA, the conclusions from these studies are derived from the assessment of either $0 \%$ or $100 \%$ editing in cell lines or as recombinant protein, as this is what can be robustly genetically engineered. The editing levels of these transcripts in vivo yield a mixed population of edited/unedited protein, leading to a more nuanced dynamic between populations of the protein. In vivo analysis of the roles of editing at these evolutionarily conserved sites, such as was undertaken for FLNA, is required to confirm their physiological importance. Rather than focus on the consequences of individual editing events, we have now generated animals completely lacking editing. Strikingly the absence of editing at all sites, including evolutionarily conserved recoding sites, was tolerated in the Adar1 ${ }^{E 861 A / E 861 A} A_{d a r b 1^{-/-}}$animals. It is important to emphasize that the analysis conducted to date has only assessed a limited number of parameters of the Adar1 ${ }^{E 861 A / E 861 A}{ }^{A d a r b 1} 1^{-/-}$animals and these have all been under standard housing conditions. It remains to be determined if functions for editing sites may only be subtle, apparent in vivo under specific conditions or when the Adar1 ${ }^{E 861 A / E 861 A} A d a r b 1^{-/-}$mice are challenged. While acknowledging that additional phenotypic differences may become apparent with further testing, the current results demonstrate that mammalian development and long-term survival can occur effectively in the absence of A-to-I editing.

By removing editing completely, we can conclude that other proposed consequences of A-to-I editing, such as miRNA recoding/retargeting $[59,60]$ and involvement in circular RNA biology [61], are not physiologically essential. Furthermore, the datasets generated herein provide a genetically controlled reference set from a single tissue and developmental timepoint for testing computational methods and will provide a resource for further understanding of A-to-I editing in vivo. The direct extrapolation of our findings to humans requires deliberation, particularly as the detailed mapping of editing across species has demonstrated that humans/primates have a significantly greater number of editing sites than rodents $[4,7,28]$. The majority of editing in humans occurs in the primate restricted $A l u$ elements. Despite the absolute numerical difference of editing between species, the genetics (MDA5 dependence) and transcriptional consequences (interferonopathy) of loss of function mutations in human $A D A R$ are highly conserved with the features of loss of Adarl, either completely or the specific inactivation of editing activity, in mouse [30, 31, 62]. The phenotypic similarity suggests that, at least for ADAR1 substrates, the loss of editing of Alu elements is not a human/primate constrained driver of innate immune activation. Rather, the genetic results indicate that the consequences of a loss of ADAR1 activity are most likely due to species conserved secondary structures formed by unedited dsRNA that can be bound by MDA5, rather than species unique substrates [35].

A-to-I editing has been postulated to be a mechanism to fine-tune and diversify the output of the genome $[1,2]$. The genetic evidence and analysis of the $\operatorname{Adar1} 1^{E 861 A / E 861 A} A$ $\operatorname{darb}^{-/-}$animals we provide indicates that mice tolerate being editing deficient surprisingly well, once MDA5mediated self-sensing of dsRNA is prevented and the single edited site within GRIA2 is provided genomically. The in vivo result demonstrates that ADAR1 and ADAR2's physiological functions are restricted to distinct pathways despite a significant fraction of editing being mediated interchangeably by either ADAR1 or ADAR2, particularly in the brain. It was particularly unexpected that strongly edited, evolutionarily conserved sites within coding regions do not appreciably affect development or lifespan of the mouse. Furthermore, as most editing occurs at a frequency of less than $20 \%$, it is likely stochastic and is not required for normal mammalian development and homeostasis. Our data do not, however, rule out the possibility of more subtle phenotypic consequences of these editing sites under certain conditions. The genetic result indicates two distinct sets of physiologically essential editing events in vivo: (a) 
the recoding of the GRIA2 Q/R site by ADAR2 and (b) the unedited transcripts that become MDA5 substrates in the absence of ADAR1-mediated editing. The identity of the substrates that become immunogenic in the absence of ADAR1-mediated editing is an open question and remains a topic of intense interest. The current hypothesis is that they are present within the hyperedited transcript population; however, the number and identity of those that are immunogenic remains to be directly shown. Collectively, these critical events likely comprise a small subset of the editome and for these essential sites ADAR1 and ADAR2 are non-redundant and do not compensate for each other. These results demonstrate that in vivo biologically essential protein recoding mediated by A-to-I editing is an exception in mammals.

\section{Conclusions}

A-to-I editing is one of the most common modifications in the mammalian transcriptome. Despite its abundance, our knowledge of the physiological functions of the vast majority of editing events is unknown. While mapping of the numbers and extent of A-to-I editing in multiple species is approaching saturation, only a handful of substrates have been characterized to date. To address this knowledge gap, we have now generated and characterized mice globally lacking A-to-I editing by crossing ADAR1-editing-deficient animals (Adar1 ${ }^{E 861 A / E 861 A} I$ fih1 ${ }^{-/-}$) with rescued Adarb $1^{-/-}$Gria $2^{R / R}$ animals. Unexpectedly, mice completely lacking A-to-I editing are strikingly normal when provided with the respective rescue alleles. The absence of additional phenotypes in the compound editing-deficient mice demonstrates that the physiologically essential functions of ADAR1 and ADAR2 do not intersect, despite a substantial degree of overlapping editing capacity by both enzymes. While Ato-I editing has long been associated with protein recoding and proteome diversification, physiologically essential protein recoding is an extremely rare (singular) event in the mouse. An implication of these findings is that a significant proportion of A-to-I editing may be stochastic and its global absence does not result in profound phenotypic consequence to a whole organism in vivo.

\section{Methods}

\section{Animals}

Adar ${ }^{E 861 A /+}$ (Adar1 ${ }^{E 861 A /+}$; MGI allele: Adar ${ }^{\text {tm1.1Xen }}$; MGI:5805648) [30], Ifih1 ${ }^{-/-}$(MGI: Ifih1 $1^{\text {tm1.1Cln }}$; MGI: 3663677) [30, 63], Adarb1 ${ }^{-/-}$(Adar2 ${ }^{-/}$; MGI: Adarb1 $^{\text {tm1.1Phs }}$; MGI:2178079) [25], and Gria2 ${ }^{R / R}$ (MGI: Gria2 ${ }^{\text {tm1.1Phs }}$; MGI:2178125) $[25,64]$ mice were on a backcrossed C57BL/6 background as previously described. Animals were housed under standard SPF conditions with food and water ad libitum. Weaning weights were recorded on the day of weaning ( 20-22 days of age). Nuclear magnetic resonance (NMR) relaxometry (EchoMRI) was performed on 12-week-old male animals of the indicated genotype, including 12-week-old male wild-type (WT) C57BL/6 animals bred and housed in the same facility, as directed by the manufacturer. For histopathology, 3 male Adar1 ${ }^{E 861 A /+}$ Ifih $^{-/-}$Adarb1 $^{+/-}$Gria2 $^{R / R}$ (dHet) and Adar1 ${ }^{\text {E861A/E861A }}$ Ifih1 $^{-/-}$Adarb1 $^{-/-}$Gria2 $^{R / R}$ $(\mathrm{dKO})$ at $\sim 20-25$ weeks of age were assessed. Tissue collection and histology was performed by the Australian Phenomics Network Histopathology and Organ Pathology Core, University of Melbourne, on tissue listed in the report. The samples were genotype blinded to the pathologists, and sections were assessed by independent pathologists. The full pathology report is available in Additional file 2: Dataset S1.

\section{Cell counts and flow cytometry analysis of peripheral blood}

Peripheral blood was analyzed on a hematological analyzer (Sysmex KX-21 N, Roche Diagnostics). Single cell suspensions from the $\mathrm{BM}$, spleen, and thymus were prepared by passing through a $23 \mathrm{G}$ needle $(\mathrm{BM})$ or crushing through a $40-\mu \mathrm{m}$ cell strainer (spleen/thymus) [38]. Antibodies against murine B220 (APC-eFluor780), CD11b/Mac1 (PE), Gr1 (PE-Cy7), F4/80 (APC), CD4 (eFluor450), and CD8 (PerCP-Cy5.5) were all obtained from eBioscience $[30,38]$. Cells were analyzed on a BD LSRIIFortessa (BD Biosciences). Results were analyzed with FlowJo software version 10.0 (Treestar).

\section{qRT-PCR and Sanger sequencing}

Total RNA was isolated from the whole brain of 12-weekold male mice of the indicated genotypes. The tissues were isolated, flash frozen in liquid nitrogen, and then homogenized in Trisure reagent using IKA T10 basic S5 Ultraturrax Disperser. RNA was extracted using Direct-Zol columns (Zymo Research) as per the manufacturer's instructions. Complementary DNA (cDNA) was synthesized using Tetro cDNA synthesis kit (Bioline) with a Htr2c-specific RT primer (5'-TGTCAACGGGATGAAG AATGCC). The previously defined edited sites in Htr2c were identified by Sanger sequencing of PCR product (not further purified or cloned) by the Australian Genome Research Facility, Melbourne (forward primer 5'-GGCCAG CACTTTCAATAGTCGTG, reverse primer 5' -CAATCT TCATGATGGCCTTAGTCC).

\section{RNA-seq samples and library preparation}

Total RNA was isolated from the whole brain from three independent biological replicates from 12-week-old male Adar1 ${ }^{\text {E861A/+ }}$ Ifih1 $^{-/-}$Adarb1 $^{+/-}$Gria2 $^{R / R}$, Adarl ${ }^{+/+}$Ifih $^{-/-}{ }^{-1}$ $\operatorname{darb1}^{-/-}$Gria2 $^{R / R}$, and Adar1 ${ }^{\text {E861A/E861A }}$ Ifih $^{-/-}$Adarb1 $^{-/-}$ $\operatorname{Gria}^{R / R}$ mice ( $n=3 /$ genotype). The tissues were isolated, flash frozen, and then homogenized in Trisure reagent 
using IKA T10 basic S5 Ultra-turrax Disperser. RNA was extracted using Direct-Zol columns (Zymo Research) as per the manufacturer's instructions. Post ribosomedepleted RNA was purified and subjected to indexing and library preparation using the Kapa Stranded RNA-seq Library Preparation Kit (Kapa Biosystems) [38] and sequenced using the Illumina platform with 150-bp pairedend reads by Novogene (Novogene (HK), Hong Kong).

\section{RNA-seq analysis}

Reads from two different technologies were used in the analysis: Adarl ${ }^{E 861 A /+}$ Ifih $^{-/-}$Adarb $^{+/-}$Gria2 $^{R / R}$ (dHet), Adar1 ${ }^{+/+}$Ifih $^{-/-}$Adarb1 $^{-/-}$Gria2 $^{R / R}$ (Adar2 ${ }^{-/-}$), and Adar1 ${ }^{E 861 A / E 861 A}$ Ifih $1^{-/}$Adarb1 ${ }^{-/-}$Gria2 $^{R / R}$ (dKO) (150-bp paired end) and the previously published

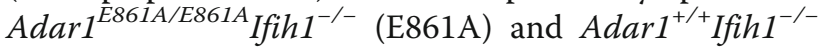
(WT) samples (GSE94387) (75 bp paired end).

\section{Pre-processing}

Sequenced reads (150 bp) were trimmed for adaptor sequence and low-quality reads using fastp (v 0.19.5) [65]. Parameters: --trim_front 10 --trim_front 210 . Sequenced reads ( $75 \mathrm{bp}$ ) from GSE94387 were trimmed for adaptor sequence and low-quality reads using (v 0.19.5) [65]. Parameters: --trim_front1 10 --trim_front2 10 --trim_tail1 1 --trim_tail2 1 . Reads mapping to rRNA were removed using Bbmap (parameters: bbsplit.sh minratio $=0.56$ minhits $=1$ maxindel $=16000)[$ BBMap Bushnell B. - sourceforge.net/projects/bbmap/].

\section{Gene expression}

For transcriptome analysis, trimmed reads were aligned using Salmon [66] (version v0.11.3) against mm10 (annotation: gencode.mm10.vM14.annotation.gtf).

Differential gene expression analysis was performed using the Degust analysis tool (http://victorian-bioinformatics-consortium.github.io/degust/). Briefly, genes were only considered with count $>3$ and CPM $>1$ in at least $3 /$ 3 samples of a given genotype. Normalized read counts (moderated log counts per million) and differential expression were generated using edgeR [67]. Each comparison (E861A vs WT, A2KO vs Het, DKO vs Het) was performed separately. See Additional file 3: Dataset S2.

\section{QuSAGE gene set testing}

Quantitative Set Analysis for Gene Expression (QuSAGE) [68] of the consensus interferon-stimulated gene (ISG)/cytokine signature defined by Liu et al. [20] was performed on using the gene expression data. See Additional file 4: Dataset S3.

\section{Editing analysis \\ Mapping}

Trimmed reads were aligned to the MM10/GRCm38 reference genome with transcript annotation (gencode.mm10.vM14.annotation.SEQINS.gtf) with STAR (version 2.6.0c) [69] using the following parameters: --outFilterType BySJout --outSAMattributes NH HI AS NM MD --outFilterMultimapNmax 20 --outFilterMismatchNmax 999 --outFilterMismatchNoverReadLmax 0.04 --alignIntronMin 20 --alignIntronMax 1000000 --alignMatesGapMax 1000000 --alignSJoverhangMin 8 --alignSJDBoverhangMin 1 --sjdbScore 1 --sjdbOverhang 149. Duplicate reads were marked Picard ["Picard Toolkit." 2019. Broad Institute, GitHub Repository. http:// broadinstitute.github.io/picard/; Broad Institute].

\section{Known sites}

A database of 57,077 murine editing sites was compiled from published databases (RADAR [8]), publications $[4,30,43]$, and unpublished murine datasets (JH-F, AMC, and CRW) and the datasets assessed for editing at these sites. Sites were marked as hyperedited if there were $>10$ editing sites within $100 \mathrm{bp}$, and no consideration was made about editing level or if editing occurred in this dataset [10]. See Additional file 5: Dataset S4A.

\section{Calling known sites}

Editing calling of known sites (RNA vs mm10) was performed using JACUSA 2.0.0-RC5 (70) (https://github. com/dieterich-lab/JACUSA): parameters used: -F 1024 -filterNH_ 99, -filterNM_ 99, -c 3 -P RF-FIRSTSTRAND. Briefly, call-1 was used to determine the RNA editing level for all known sites for each individual sample replicate. Duplicate reads were removed. For sites not called by JACUSA, we added read depth calculated by samtools pileup to reflect the sequence coverage at those positions. The editing rate for each genotype was calculated as the sum of edited reads for three replicates/total read depth for all three replicates. Sites required $\geq 50$ read coverage in all samples (a combined read coverage of $\geq 50$ for all genotypes was required) of the comparison and an editing rate of $\geq 0.01(\geq 1 \%)$ in the WT and dHet to be considered. See Additional file 5: Dataset S4A.

\section{Differential editing of known sites}

Calling of differential editing in known sites across genotypes was performed using JACUSA 2.0.0-RC5 [70]. Briefly, call-2 was used to determine the difference in editing level for all known sites (all replicates of genotype A vs all replicates of genotype B). Duplicate reads were removed. Sites required $\geq 50$ read coverage and an editing rate of $\geq 0.01(\geq 1 \%)$ to be considered. See Additional file 5: Dataset S4A. 


\section{Calling novel editing sites}

Editing calling of novel sites was performed using JACUSA 2.0.0-RC5 [70]. Briefly, call-2 was used to determine the RNA editing level for all sites within $5 \mathrm{~kb}$ of all Ensembl genes (ensembl_genes_96) using all replicates for each genotype. Duplicate reads were removed, and min coverage of 3 per sample (parametres used: -F 1024 -filterNH_99, -filterNM_99, -c 3 -P RF-FIRSTSTRAND). A site was considered edited if score $\mathrm{z}>5$; all sites with score $\mathrm{z}>5$ in the DKO were removed after manual assessment. See Additional file 6: Dataset S4B.

\section{Annotation}

Editing sites were annotated with gene, gene part (promoter, Exon, intron, 3' UTR, or intergenic) using Goldmine [71]. B1 and B2 SINE annotation (mm10) was from UCSC rmsk table [https://www.ncbi.nlm.nih.gov/ pubmed/23155063]. See Additional files 5, 6, and 7: Datasets S4A, S4B, S5.

\section{Sequence logos}

Sequence logos were generated using ggseqlogo [72].

\section{Datasets}

All datasets related to this work are deposited in GEO. Dataset accession number: GSE132214 (https://www. ncbi.nlm.nih.gov/geo/query/acc.cgi?acc=GSE132214).

\section{Statistical analysis}

For biological experiments, the significance of results was analyzed using the one-way or two-way ANOVA with multiple comparison corrections unless otherwise stated; calculated as Prism software, $P<0.05$ was considered significant. Data are presented as mean \pm SEM unless otherwise stated.

\section{Supplementary information}

Supplementary information accompanies this paper at https://doi.org/10. 1186/s13059-019-1873-2.

\footnotetext{
Additional file 1: Figure S1 (related to Figure 3). Comparison of gene expression signatures by genotype; data from Panel 3A. Analysis of transcriptional signatures in the 12 week old male brain of each genotype. $n=3$ independent samples per genotype. The increased expression of the transcripts highlighted in blue is shared between murine and human ADAR1 mutants. (A) Y-axis has the gene expression comparison of the Adar2-/- vs the dHet; $\mathrm{x}$-axis has the gene expression comparison of the Adar1E861A/E861A vs WT. Gene expression changes dependent on Adar1 loss occur on the $x$ axis, those dependent on the loss of Adar2 on the $y$ axis. (B) Adar2-/-compared to Adar1E861A/E861A Adar2-/- (dKO); (C) Adar1E861A/E861A compared to Adar1E861A/E861A Adar2-/- (dKO). Figure S2. Comparison of the gene expression signatures by genotypes; data derived from comparisons in Panel 2A. Figure S3 (related to Figure 4). Altered sites identified in analysis of Adar1E861A E861A Adarb1-/- (dKO); related to Panel 4B. Analysis of sites identified as altered compared to ref seq or batch control in the dKO samples. Individual sites with IGV screenshots and the full list of sites with variants identified in analysis of the double $\mathrm{KO}$ samples.
}

Additional file 2 Dataset S1. Full histopathology report from Adar1E861A/+Ifih1-/-Adarb1+/-Gria2R/R (dHet) and Adar1E861A/ E861Alfih1-/-Adarb1-/-Gria2R/R (dKO).

Additional file 3: Dataset S2. RNA-seq data used for differential gene expression analysis. Samples $=12$ week old male whole brain; $n=3$ per genotype. Related to Fig 2 and Fig S2.

Additional file 4: Dataset S3. QUSAGE pathway analysis of gene expression datasets. Samples $=12$ week old male whole brain; $n=3$ per genotype. Related to Fig 2 and Fig S2.

Additional file 5: Dataset S4A. Editing analysis of the known sites. Related to Fig. 3 and Fig. 4.

Additional file 6: Dataset S4B. De novo discovery of RNA editing sites in each genotype using JACUSA2.0.0 (transcriptome comparison to C57BI/6 reference genome). Related to Fig 3 and Fig 4.

Additional file 7: Dataset S5. ADAR1 and ADAR2 specific editing events - frequency of editing. Related to Fig. 4C.

Additional file 8: Review history.

\section{Acknowledgements}

The authors thank JB Li, L Purton, and J Heierhorst for the comments and discussion; C Dieterich for the access to JACUSA 2; P Seeburg, M Higuchi, K Matthaei, and the Australian Phenomics Facility PhenomeBank for providing the Adarb $1^{-1-}$ Gria2 $2^{R / R}$ animals; St. Vincent's Hospital BioResources Centre staff for care of experimental animals; St. Vincent's Institute Flow Cytometry Core Facility; and APN Histopathology and Organ Pathology Service, University of Melbourne.

\section{Review history}

The review history is available as Additional file 8.

\section{Peer review information}

Barbara Cheifet was the primary editor of this article and managed its editorial process and peer review in collaboration with the rest of the editorial team.

\section{Authors' contributions}

$\mathrm{JH}-\mathrm{F}$ and CRW were responsible for the conceptualization; JH-F, AMC, and CRW were responsible for the methodology; JH-F, AMC, ST, and CRW performed the experiments and data analysis; JH-F and CRW wrote the original draft; JH-F, AMC, ST, and CRW reviewed and edited the manuscript; $\mathrm{JH}-\mathrm{F}$ and CRW were responsible for the funding acquisition; and $\mathrm{JH}-\mathrm{F}$ and $\mathrm{CRW}$ provided supervision. All authors read and approved the final manuscript.

\section{Funding}

This work was supported by the National Health and Medical Research Council, Australia (NHMRC; CRW and JB Li, APP1102006/APP1144049); Australian Research Council (CRW; DP180103989); Victorian Cancer Agency Research Fellowship (CRW; MCRF15015); Marian and E.H. Flack Trust (JH-F); SVI Women in Science Award $(\mathrm{JH}-\mathrm{F})$; and in part by the Victorian State Government Operational Infrastructure Support (to St Vincent's Institute).

\section{Availability of data and materials}

All datasets described in this work are deposited in GEO under accession code GSE132214 [73]. Mouse strains are available from the Australian Phenome Bank (https://pb.apf.edu.au/phenbank/homePage.html).

Ethics approval and consent to participate

All animal experiments were approved by the Animal Ethics Committee of St. Vincent's Hospital, Melbourne (AEC\#031/15 and AEC\#009/18).

Consent for publication

Not applicable.

Competing interests

The authors declare that they have no competing interests. 


\section{Author details}

'St. Vincent's Institute of Medical Research, 9 Princes St, Fitzroy, VIC 3065, Australia. ${ }^{2}$ Department of Medicine, St. Vincent's Hospital, Melbourne Medical School, University of Melbourne, Fitzroy, VIC 3065, Australia. ${ }^{3}$ Mary MacKillop Institute for Health Research, Australian Catholic University, Melbourne, VIC 3000, Australia.

Received: 29 July 2019 Accepted: 29 October 2019

Published online: 09 December 2019

\section{References}

1. Bass BL. RNA editing by adenosine deaminases that act on RNA. Annu Rev Biochem. 2002;71(1):817-46.

2. Eisenberg E, Levanon EY. A-to-I RNA editing - immune protector and transcriptome diversifier. Nat Rev Genet. 2018;19(8):473-90.

3. Walkley CR, Li JB. Rewriting the transcriptome: adenosine-to-inosine RNA editing by ADARs. Genome Biol. 2017;18(1):205.

4. Tan MH, Li Q, Shanmugam R, Piskol R, Kohler J, Young AN, et al. Dynamic landscape and regulation of RNA editing in mammals. Nature. 2017; 550(7675):249-54.

5. Polson AG, Bass BL. Preferential selection of adenosines for modification by double-stranded RNA adenosine deaminase. EMBO J. 1994;13(23):5701-11.

6. Solomon O, Di Segni A, Cesarkas K, Porath HT, Marcu-Malina V, Mizrahi O, et al. RNA editing by ADAR1 leads to context-dependent transcriptomewide changes in RNA secondary structure. Nat Commun. 2017:8(1):1440.

7. Bazak L, Haviv A, Barak M, Jacob-Hirsch J, Deng P, Zhang R, et al. A-to-I RNA editing occurs at over a hundred million genomic sites, located in a majority of human genes. Genome Res. 2014;24(3):365-76.

8. Ramaswami G, Li JB. RADAR: a rigorously annotated database of A-to-I RNA editing. Nucleic Acids Res. 2014;42(1):D109-13.

9. Porath $H T$, Knisbacher BA, Eisenberg E, Levanon EY. Massive A-to-I RNA editing is common across the Metazoa and correlates with dsRNA abundance. Genome Biol. 2017;18(1):185.

10. Porath HT, Carmi S, Levanon EY. A genome-wide map of hyper-edited RNA reveals numerous new sites. Nat Commun. 2014:5:4726.

11. Whipple JM, Youssef OA, Aruscavage PJ, Nix DA, Hong C, Johnson WE, et al. Genome-wide profiling of the C. elegans dsRNAome. RNA. 2015; 21(5):786-800

12. Blango MG, Bass BL. Identification of the long, edited dsRNAome of LPSstimulated immune cells. Genome Res. 2016;26(6):852-62.

13. Mladenova D, Barry G, Konen LM, Pineda SS, Guennewig B, Avesson L, et al. Adar3 is involved in learning and memory in mice. Front Neurosci. 2018;12:243

14. Melcher T, Maas S, Herb A, Sprengel R, Higuchi M, Seeburg PH. RED2, a brain-specific member of the RNA-specific adenosine deaminase family. J Biol Chem. 1996:271(50):31795-8.

15. Rice Gl, Kitabayashi N, Barth M, Briggs TA, Burton ACE, Carpanelli ML, et al. Genetic, phenotypic, and interferon biomarker status in ADAR1-related neurological disease. Neuropediatrics. 2017;48(3):166-84.

16. Hartner JC, Walkley CR, Lu J, Orkin SH. ADAR1 is essential for the maintenance of hematopoiesis and suppression of interferon signaling. Nat Immunol. 2009:10(1):109-15.

17. Crow YJ, Manel N. Aicardi-Goutieres syndrome and the type I interferonopathies. Nat Rev Immunol. 2015;15(7):429-40.

18. Han L, Diao L, Yu S, Xu X, Li J, Zhang R, et al. The genomic landscape and clinical relevance of A-to-I RNA editing in human cancers. Cancer Cell. 2015; 28(4):515-28.

19. Peng $X$, Xu X, Wang $Y$, Hawke DH, Yu S, Han L, et al. A-to-l RNA editing contributes to proteomic diversity in cancer. Cancer Cell. 2018;33(5):817-28 e7.

20. Liu H, Golji J, Brodeur LK, Chung FS, Chen JT. deBeaumont RS, et al. Tumorderived IFN triggers chronic pathway agonism and sensitivity to ADAR loss. Nat Med. 2019;25(1):95-102.

21. Ishizuka JJ, Manguso RT, Cheruiyot CK, Bi K, Panda A, Iracheta-Vellve A, et al. Loss of ADAR1 in tumours overcomes resistance to immune checkpoint blockade. Nature. 2019;565(7737):43-8.

22. Gannon HS, Zou T, Kiessling MK, Gao GF, Cai D, Choi PS, et al. Identification of ADAR1 adenosine deaminase dependency in a subset of cancer cells. Nat Commun. 2018;9(1):5450

23. Gallo A, Vukic D, Michalik D, O'Connell MA, Keegan LP. ADAR RNA editing in human disease; more to it than meets the I. Hum Genet. 2017;136(9):1265-78.
24. Tran SS, Jun HI, Bahn JH, Azghadi A, Ramaswami G, Van Nostrand EL, et al. Widespread RNA editing dysregulation in brains from autistic individuals. Nat Neurosci. 2019;22(1):25-36.

25. Higuchi M, Maas S, Single FN, Hartner J, Rozov A, Burnashev N, et al. Point mutation in an AMPA receptor gene rescues lethality in mice deficient in the RNA-editing enzyme ADAR2. Nature. 2000;406(6791):78-81.

26. Horsch M, Seeburg PH, Adler T, Aguilar-Pimentel JA, Becker L, Calzada-Wack $J$, et al. Requirement of the RNA-editing enzyme ADAR2 for normal physiology in mice. J Biol Chem. 2011;286(21):18614-22.

27. Terajima H, Yoshitane H, Ozaki H, Suzuki Y, Shimba S, Kuroda S, et al. ADARB1 catalyzes circadian A-to-l editing and regulates RNA rhythm. Nat Genet. 2017;49(1):146-51.

28. Zhang R, Deng P, Jacobson D, Li JB. Evolutionary analysis reveals regulatory and functional landscape of coding and non-coding RNA editing. PLOS Genet. 2017;13(2):e1006563.

29. Pinto Y, Cohen HY, Levanon EY. Mammalian conserved ADAR targets comprise only a small fragment of the human editosome. Genome Biol. 2014;15(1):R5.

30. Liddicoat BJ, Piskol R, Chalk AM, Ramaswami G, Higuchi M, Hartner JC, et al. RNA editing by ADAR1 prevents MDA5 sensing of endogenous dsRNA as nonself. Science. 2015:349(6252):1115-20.

31. Pestal K, Funk CC, Snyder JM, Price ND, Treuting PM, Stetson DB. Isoforms of RNAediting enzyme ADAR1 independently control nucleic acid sensor MDA5-driven autoimmunity and multi-organ development. Immunity. 2015;43(5):933-44.

32. Mannion NM, Greenwood SM, Young R, Cox S, Brindle J, Read D, et al. The RNA-editing enzyme ADAR1 controls innate immune responses to RNA. Cell Rep. 2014;9(4):1482-94.

33. George CX, Ramaswami G, Li JB, Samuel CE. Editing of cellular self-RNAs by adenosine deaminase ADAR1 suppresses innate immune stress responses. J Biol Chem. 2016;291(12):6158-68.

34. Chung H, Calis JJA, Wu X, Sun T, Yu Y, Sarbanes SL, et al. Human ADAR1 prevents endogenous RNA from triggering translational shutdown. Cell. 2018;172(4):811-24 e14.

35. Pfaller CK, Donohue RC, Nersisyan S, Brodsky L, Cattaneo R. Extensive editing of cellular and viral double-stranded RNA structures accounts for innate immunity suppression and the proviral activity of ADAR1p150. PLoS Biol. 2018;16(11):e2006577.

36. Wang Q, Miyakoda M, Yang W, Khillan J, Stachura DL, Weiss MJ, et al. Stressinduced apoptosis associated with null mutation of ADAR1 RNA editing deaminase gene. J Biol Chem. 2004;279(6):4952-61.

37. Hartner JC, Schmittwolf C, Kispert A, Muller AM, Higuchi M, Seeburg PH. Liver disintegration in the mouse embryo caused by deficiency in the RNAediting enzyme ADAR1. J Biol Chem. 2004;279(6):4894-902.

38. Heraud-Farlow JE, Chalk AM, Linder SE, Li Q, Taylor S, White JM, et al. Protein recoding by ADAR1-mediated RNA editing is not essential for normal development and homeostasis. Genome Biol. 2017;18(1):166.

39. Eggington JM, Greene T, Bass BL. Predicting sites of ADAR editing in double-stranded RNA. Nat Commun. 2011;2:319.

40. Lehmann KA, Bass BL. Double-stranded RNA adenosine deaminases ADAR and ADAR2 have overlapping specificities. Biochemistry. 2000;39(42):12875-84.

41. Merkle T, Merz S, Reautschnig P, Blaha A, Li Q, Vogel P, et al. Precise RNA editing by recruiting endogenous ADARs with antisense oligonucleotides. Nat Biotechnol. 2019;37(2):133-8.

42. Qu L, Yi Z, Zhu S, Wang C, Cao Z. Zhou Z, et al. Nat Biotechnol: Programmable RNA editing by recruiting endogenous ADAR using engineered RNAs; 2019.

43. Liddicoat BJ, Hartner JC, Piskol R, Ramaswami G, Chalk AM, Kingsley PD, et al. Adenosine-to-inosine RNA editing by ADAR1 is essential for normal murine erythropoiesis. Exp Hematol. 2016;44(10):947-63.

44. Riedmann EM, Schopoff S, Hartner JC, Jantsch MF. Specificity of ADAR-mediated RNA editing in newly identified targets. RNA. 2008;14(6):1110-8.

45. Washburn MC, Kakaradov B, Sundararaman B, Wheeler E, Hoon S, Yeo GW et al. The dsRBP and inactive editor ADR-1 utilizes dsRNA binding to regulate A-to-I RNA editing across the C. elegans transcriptome. Cell Rep. 2014;6(4):599-607.

46. Licht K, Kapoor U, Amman F, Picardi E, Martin D, Bajad P, et al. A high resolution A-to-l editing map in the mouse identifies editing events controlled by pre-mRNA splicing. Genome Res. 2019;29(9):1453-63.

47. Ramaswami G, Deng P, Zhang R, Anna Carbone M, Mackay TFC, Billy LJ. Genetic mapping uncovers cis-regulatory landscape of RNA editing. Nat Commun. 2015;6:8194. 
48. Deffit SN, Yee BA, Manning AC, Rajendren S, Vadlamani P, Wheeler EC, et al. The $C$. elegans neural editome reveals an ADAR target mRNA required for proper chemotaxis. Elife. 2017;6.

49. Tonkin LA, Saccomanno L, Morse DP, Brodigan T, Krause M, Bass BL. RNA editing by ADARs is important for normal behavior in Caenorhabditis elegans. EMBO J. 2002;21(22):6025-35.

50. Palladino MJ, Keegan LP, O'Connell MA, Reenan RA. A-to-I pre-mRNA editing in Drosophila is primarily involved in adult nervous system function and integrity. Cell. 2000;102(4):437-49.

51. Keegan LP, McGurk L, Palavicini JP, Brindle J, Paro S, Li X, et al. Functional conservation in human and Drosophila of Metazoan ADAR2 involved in RNA editing: loss of ADAR1 in insects. Nucleic Acids Res. 2011:39(16):7249-62.

52. Grice LF, Degnan BM. The origin of the ADAR gene family and animal RNA editing. BMC Evol Biol. 2015;15:4.

53. Garrett $\mathrm{S}$, Rosenthal JJ. RNA editing underlies temperature adaptation in K+ channels from polar octopuses. Science. 2012;335(6070):848-51.

54. Liscovitch-Brauer N, Alon S, Porath HT, Elstein B, Unger R, Ziv T, et al. Tradeoff between transcriptome plasticity and genome evolution in cephalopods. Cell. 2017;169(2):191-202 e11.

55. Higuchi $M$, Single FN, Kohler M, Sommer B, Sprengel R, Seeburg PH. RNA editing of AMPA receptor subunit GluR-B: a base-paired intron-exon structure determines position and efficiency. Cell. 1993;75(7):1361-70.

56. Jain M, Mann TD, Stulic M, Rao SP, Kirsch A, Pullirsch D, et al. RNA editing of Filamin A pre-mRNA regulates vascular contraction and diastolic blood pressure. EMBO J. 2018;37(19). https://doi.org/10.15252/embj.201694813.

57. Yeo J, Goodman RA, Schirle NT, David SS, Beal PA. RNA editing changes the lesion specificity for the DNA repair enzyme NEIL1. Proc Natl Acad Sci U S A. 2010;107(48):20715-9.

58. Chen L, Li Y, Lin CH, Chan TH, Chow RK, Song Y, et al. Recoding RNA editing of AZIN1 predisposes to hepatocellular carcinoma. Nat Med. 2013; 19(2):209-16.

59. Vesely C, Tauber S, Sedlazeck FJ, von Haeseler A, Jantsch MF. Adenosine deaminases that act on RNA induce reproducible changes in abundance and sequence of embryonic miRNAs. Genome Res. 2012;22(8):1468-76.

60. Kawahara $Y$, Zinshteyn $B$, Sethupathy $P$, lizasa $H$, Hatzigeorgiou AG, Nishikura K. Redirection of silencing targets by adenosine-to-inosine editing of miRNAs. Science. 2007:315(5815):1137-40

61. Ivanov A, Memczak S, Wyler E, Torti F, Porath HT, Orejuela MR, et al. Analysis of intron sequences reveals hallmarks of circular RNA biogenesis in animals. Cell Rep. 2015;10(2):170-7.

62. Rice GI, Kasher PR, Forte GM, Mannion NM, Greenwood SM, Szynkiewicz M, et al. Mutations in ADAR1 cause Aicardi-Goutieres syndrome associated with a type I interferon signature. Nat Genet. 2012;44(11):1243-8.

63. Gitlin L, Barchet W, Gilfillan S, Cella M, Beutler B, Flavell RA, et al. Essential role of mda-5 in type I IFN responses to polyriboinosinic:polyribocytidylic acid and encephalomyocarditis picornavirus. Proc Natl Acad Sci U S A. 2006; 103(22):8459-64.

64. Kask K, Zamanillo D, Rozov A, Burnashev N, Sprengel R, Seeburg PH. The AMPA receptor subunit GluR-B in its $Q / R$ site-unedited form is not essential for brain development and function. Proc Natl Acad Sci U S A 1998;95(23):13777-82.

65. Chen S, Zhou Y, Chen Y, Gu J. fastp: an ultra-fast all-in-one FASTQ preprocessor. Bioinformatics. 2018;34(17):i884-i90.

66. Patro R, Duggal G, Love MI, Irizarry RA, Kingsford C. Salmon provides fast and bias-aware quantification of transcript expression. Nat Methods. 2017; 14(4):417-9.

67. Robinson MD, McCarthy DJ, Smyth GK. edgeR: a Bioconductor package for differential expression analysis of digital gene expression data. Bioinformatics. 2010;26(1):139-40.

68. Yaari G, Bolen CR, Thakar J, Kleinstein SH. Quantitative set analysis for gene expression: a method to quantify gene set differential expression including gene-gene correlations. Nucleic Acids Res. 2013;41(18):e170.

69. Dobin A, Davis CA, Schlesinger F, Drenkow J, Zaleski C, Jha S, et al. STAR: ultrafast universal RNA-seq aligner. Bioinformatics. 2013;29(1):15-21.

70. Piechotta M, Wyler E, Ohler U, Landthaler M, Dieterich C. JACUSA: sitespecific identification of RNA editing events from replicate sequencing data. BMC Bioinformatics. 2017;18(1):7.

71. Bhasin JM, Ting AH. Goldmine integrates information placing genomic ranges into meaningful biological contexts. Nucleic Acids Res. 2016; 44(12):5550-6.
72. Wagih O. ggseqlogo: a versatile R package for drawing sequence logos. Bioinformatics. 2017:33(22):3645-7.

73. Chalk AC, Taylor S, Heraud-Farlow J, Walkley CR. The majority of A-to-I RNA editing is not required for mammalian homeostasis. RNA-seg datasets from 12 week old adult brain. Gene Expression Omnibus: GSE132214 (https:// www.ncbi.nlm.nih.gov/geo/query/acc.cgi?acc=GSE132214).

\section{Publisher's Note}

Springer Nature remains neutral with regard to jurisdictional claims in published maps and institutional affiliations.
Ready to submit your research? Choose BMC and benefit from:

- fast, convenient online submission

- thorough peer review by experienced researchers in your field

- rapid publication on acceptance

- support for research data, including large and complex data types

- gold Open Access which fosters wider collaboration and increased citations

- maximum visibility for your research: over $100 \mathrm{M}$ website views per year

At BMC, research is always in progress.

Learn more biomedcentral.com/submissions 